\title{
Gene Therapy for Bone Regeneration using Non-viral BMP Gene Expression Vector and in vivo Electroporation
}

\author{
Mariko Kawai, Kiyoshi Ohura \\ ${ }^{1}$ Department of Pharmacology/Osaka Dental University \\ 8-1 Kuzuha-hanazono-cho, Hirakata city, Japan \\ kawai-m@cc.osaka-dent.ac.jp; ohura@cc.osaka-dent.ac.jp
}

\begin{abstract}
It is well known that bone morphogenetic protein (BMP) induces ectopic bone formation when the recombinant protein or BMP gene is transferred into the skeletal muscle. We developed a novel method for BMP gene transfer, which is combination with nonviral BMP gene expression vector and in vivo electroporation. Then, we applied this method to transfer BMP-2 gene into the skeletal muscles of rats and induced the ectopic bone formation in the target sites. We injected BMP gene expression vector, pCAGGS-BMP-2, in the skeletal muscles of rats and immediately electroporated under the conditions of 100 voltage, 50 msec., and 8 pulses. We found the ectopic bone formation in the skeletal muscles 21 days after BMP gene transfer. In the BMP family, BMP-2/4 or BMP-2/7 heterodimer has stronger potential for bone induction compared with BMP-2, BMP-4 or BMP-7 homodimer. Then, we constructed BMP-2/7 heterodimer produced vector: pCAGGS-BMP-2/7. It has no IRES site, therefore each of BMP-2 and BMP-7 gene expression is equal. When we injected pCAGGS-BMP-2/7 plasmid vector in the skeletal muscles and immediately performed in vivo electroporation, the ectopic bone formation was induced quickly on 10 days after gene transfer. For clinical application, we need more safe procedure on in vivo electroporation under the condition of lower voltage than 100 voltage. If we set the condition: 50 voltage and 8 pulses, the efficiency of gene transfer also reduced. But, when we induced pulse number, it recovered. We evaluated proper voltage and pulse number as the same gene transfer efficiency of 100 voltage. We tried to apply this gene transfer system for alveolar bone regeneration under the condition less 50 voltage. We often use bone prosthetic material and autogenous bone graft for alveolar bone defect caused by periodontal disease or trauma. But, these therapies sometimes have some risk for patients such as infection or fractures. Our developed gene therapy system for bone regeneration will be with more safety and with less burden on the patient.
\end{abstract}

Keywords: gene therapy, non-viral vector, in vivo electroporation, BMP

\section{Introduction}

BMP has strong potential to induce ectopic bone formation in the skeletal muscles when it is transplanted in the muscles [1, 2]. For quarter century since recombinant human BMP (rhBMP) is constructed [3], rhBMP protein is not used for clinical application frequently $[4,5]$. Because rhBMP-2 protein is soluble and disperses soon after implantation, many researchers have used a matrix to retain the protein at the target site [6,7]. Gene-based delivery methods are generally divided into two categories: viral vectors and non-viral vectors [8,9]. Although adenoviral vectors are highly efficient for delivering BMP gene to the target muscles, there are several problems including the elicitation of an immune response in the host [10]. Therefore, we needed the treatment of immunosuppression by general or local to induce the ectopic bone formation in the skeletal muscles by the injection of BMP-2 gene expression adenoviral vector [11]. On the other hand, non-viral delivery methods (naked DNA, lipoplex, polyplex, and electroporation) do not need immunosuppression for successful gene delivery [10]. Electroporation can especially more rapidly express a target gene than virus-mediated gene transfer, and it can increase the transfection rate by more than 100-fold compared to the direct introduction of naked DNA [12]. In addition, gene therapy by in vivo electroporation is a simple and inexpensive method that only requires a plasmid and a device for performing electroporation. Therefore, we developed non-viral BMPs gene expression vector and tried to induce bone formation using in vivo electroporation [13]. BMPs family has several subtypes with high osteoinductive potential, and especially, BMP heterodimers as BMP-2/4 or BMP-2/7 have greater osteogenic potential than BMP homodimers [14, 15]. Then, we constructed BMP-2/BMP-7 heterodimer produced non-viral gene expression vector: pCAGGS-BMP-2/7 and it induced ectopic bone formation in the skeletal muscles rapidly [16]. In vivo electroporation for clinical use requires safe condition under the lower voltage. Then, we searched lower voltage condition of electroporation for bone induction in the skeletal muscles keeping same gene transferred efficiency [17]. In this study, we tried to gene transfer using non-viral BMP-2/7 gene 
expression vector and in vivo electroporation under more safer conditions for clinical use on the goal of the alveolar bone regeneration.

\section{Material and Methods}

First, we tried to search safer and lower voltage for the rat mucosa as the gene transferred target areas. We incised the skin of lower limbs and injected pCAGGS-lacZ $(0.5 \mu \mathrm{g} / \mu \mathrm{l})$ and immediately performed in vivo electroporation under several lower voltages less than 50 voltage. After 24 hours, we took lower limbs and measured X-gal staining of homogenized samples. The samples were measured by $420 \mathrm{~nm}$. Next, we made the electrodes for the oral areas of the nine weeks old male Wistar rats using the system of dental impression (Fig. 1). Then, we tried to gene transfer to the periodontal tissues of rats using non-viral vectors; pCAGGS-GFP and performed in vivo electroporation under low voltage conditions. After gene transfer, we made histological samples and analyzed histological changes.

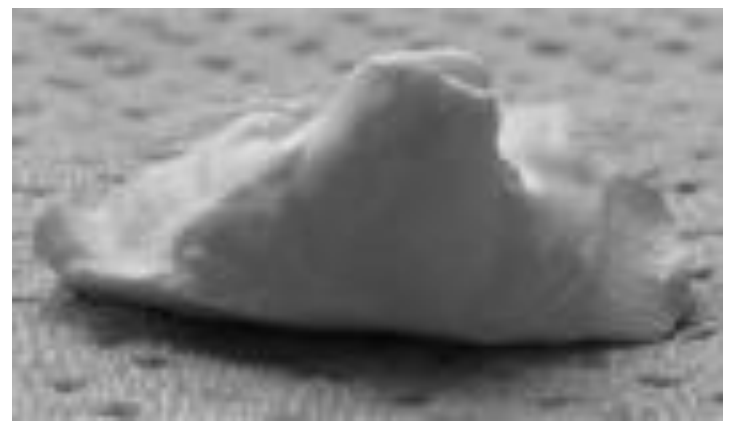

Fig. 1: Making electrodes for rats.

\section{Results}

We could performe gene transfer and in vivo electroporation to the maxillary periodontal tissues of rats using hand made original electrodes by the dental impression system. If the incision was not performed, it was not possible to recover the efficiency of the gene transfer under less 50 voltage condition even though the loading time or pulse numbers increased (Fig.2). But, the skin incision was procedured and we directly performed in vivo electroporation to the target of mucosa, the efficiency of gene transfer recovered as the condition of 100 voltage, $50 \mathrm{msec}$. and 8 pulses (Fig.2). One day after GFP gene transfer to the periodontal tissues of rats, we found GFP positive cells in the target areas (Fig.3).

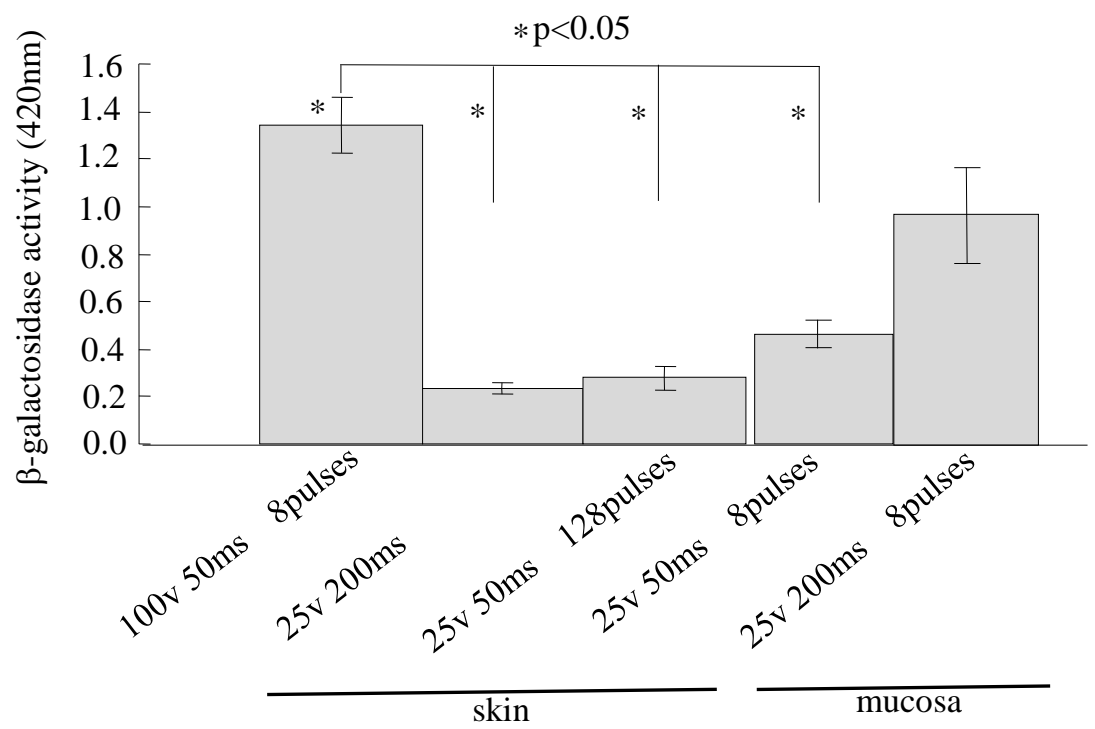

Fig. 2: The efficiency of gene transfer under the lower voltages. 


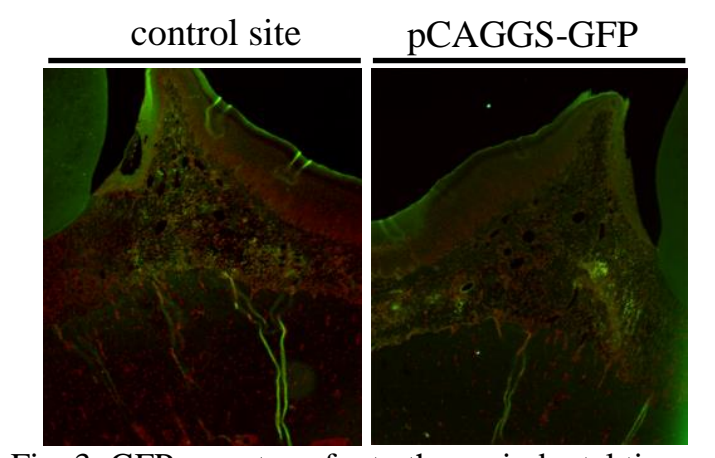

Fig. 3: GFP gene transfer to the periodontal tissues.

\section{Discussion}

We could successfully performed gene transfer using in vivo electroporation under the condition of less 100 Voltage. In this time, we transferred GFP gene expression vector to the maxillary periodontal tissues of rats under the condition of 50 voltage, $50 \mathrm{msec}$. and 32 pulses. One day after GFP gene transfer using in vivo electroporation, the GFP positive cells were found in the areas of target site in the periodontal tissues of rats. On the other hands, we could not find GFP positive cells in the control site. For future clinical use, less 50 voltage condition for in vivo electroporation would be more safe and better. Therefore, we tried to achieve same level of the efficiency as the condition with 100 voltage, 50msec. and 32 pulses. When we performed in vivo electroporation with non-viral LaZ expression vector under the condition with 25 voltage, $200 \mathrm{msec}$. 8 pulses, and the efficiency of gene transfer could be achieved at the same level of 100 voltage. The 25 voltage condition is almost same level with transcutaneous electric neural stimulation treatment for temporomandibular joint disorder [18, 19]. Therefore, we could try gene transfer to the oral areas using in vivo electroporation under the condition with 25 voltage, 200 msec. and 8 pulses in the next stage for alveolar bone regeneration.

\section{Conclusion}

We successfully performed gene transfer to the oral areas using non-viral vector and in vivo electroporation. It is safe and simple methods. Therefore, the combination of non-viral gene expression vector and in vivo electroporation could be expected for bone regeneration therapy for clinical application in the near future.

\section{Acknowledgements}

This study was supported in part by a Grant - in Aid Scientific Research on Innovated Areas (B). We greatly appreciate Tomoko Yamamoto, the manager in the department of animal experiments in Dental School, Okayama University.

\section{References}

[1] E. A. Wang, V. Rosen, J. S. D'Alessandro, and M. Bauduy, "Recombinant human bone morphogenetic protein induces bone formation,” in Proc. Natl. Acad. Sci. U. S. A., vol. 87, pp. 2220-2224, 1990.

[2] K. D. Hankenson, K. Gagne, and M. Shaughnessy, "Extracellular signalling molecules to promote fracture healing and bone regeneration," Adv. Drug. Deliv. Rev., vol. 94, pp. 3-12, 2015.

[3] J. M. Wozney, V. Rosen, A. J. Celeste, L. M. Mitsock, and M. J. Whitters, "Novel regulators of bone morphogenetic protein: Molecular clones and activities," Science, vol. 242, pp. 1528-1534, 1988.

[4] A. Noshchenko, L. Hoffecker, E. M. Lindley, E. L. Burger, C. M. Cain, and V. V. Patel, "Perioperative and long-term clinical outcomes for bone morphogenetic protein versus iliac crest bone graft for lumbar fusion in degenerative disk disease: systematic review with meta-analysis," J. Spinal Disord. Tech., vol. 27, no. 3, pp. 117-135, 2014.

[5] A. W. James, G. LaChaud, J. Shen, G. Asatrian, V. Nguyen, X. Zhang, K. Ting, and C. Soo, "A review of the clinical side effect of bone morphogenetic protein-2," Tissue Eng Part B Rev., vol. 22, no. 2, 2016.

[6] K. Bessho, D. L. Carnes, R. Cabin, and J. L. Ong, "Experimental studies on bone induction using low molecular weight poly (DL-lactode-co-glycolide) as a carrier fir recombinant human bone morphogenetic protein-2," J. Biomed. Mater. Res., vol. 61, pp. 61-65, 2002. 
[7] C. A. Tannoury and H. S. An, "Complications with the use of bone morphogenetic protein 2 (BMP-2) in spine surgery," Spine J., vol. 14, no. 3, pp. 552-559, 2014.

[8] T. D. Alden, P. Varady, D. F. Kallmes, J. A. Jane, and G. A. Helm, "Bone Morphogenetic protein gene therapy," Spin, vol. 27, pp. 87-93, 2002.

[9] T. Ogura, "In vivo electroporation: A new frontier for gene delivery and embryology," Differentiation, vol. 70, pp. 163$171,2002$.

[10] S. Chira, C. S. Jackson, I. Oprea, F. Ozturk, M. S. Pepper, I. Diaconu, C. Braicu, L. Z. Raduly, G. A. Calin, and I. Berindan-Neagoe, "Progresses towards safe and efficient gene therapy vectors," Oncotarget, vol. 6, no. 31, pp. 3067530703, 2015.

[11] S. Kaihara, K. Bessho, Y. Ohkubo, J. Sonobe, M. Kawai, and T. Iizuka, "Simple and effective osteoinductive gene therapy by local injection of a bone morphogenetic protein-2-expressing recombinant adenoviral vector and FK506 mixture in rats," gene therapy, vol. 11, no. 5, pp. 439-447, 2004.

[12] M. Nishikawa and L. Huang, "Non-viral vectors in the new millennium: Delivery barriers in gene transfer," human gene therapy, vol. 12, pp. 861-870, 2001.

[13] M. Kawai, K. Bessho, S. Kaihara, J. Sonobe, K. Oda, T. Iizuka, and H. Maruyama, "Ectopic bone formation by human bone morphogenetic protein-2 gene transfer to skeletal muscle using transcutaneous electroporation," human gene therapy, vol. 14, pp. 1547-1556, 2003.

[14] D. I. Isrel, J. Nove, K. M. Kerms, R. J. Kaufman, V. Rosen, K. A. Cox, and J. M. Wozney, "Heterodimers bone morihogenetic protein show enhanced activities in vitro and in vivo," Growth Factor, vol. 13, pp. 291-300, 1996.

[15] T. Morimoto, T. Kaito, Y. Matsuo, T. Sugiura, M. Kashii, M. Iwasaki, and H. Yoshikawa, "The bone morphogenetic protein-2/7 heterodimer is a stronger inducer of bone regeneration than the individual homodimers in a rat spinal fusion model," Spine J., vol. 15, no. 6, pp. 1379-1390, 2015.

[16] M. Kawai, H. Marumaya, H. Yamamoto, K. Bessho, J. Miyazaki, and T. Yamamoto, "Simple strategy for bone regeneration with a BMP-2/7 gene expression cassette vector," BBRC, vol. 390, pp. 1012-1017, 2009.

[17] H. Yamamoto, M. Kawai, N. Shiotsu, M. Watanabe, Y. Yoshida, K. Suzuki, H. Maruyama, J. Miyazaki, M. Ikegame, and T. Yamamoto, "BMP-2 gene transfer under various conditions with in vivo electroporation and bone induction," A. J. Oral Maxillo. Surg., vol. 24, pp. 49-53, 2012.

[18] J. Boever and W. D. McCall, "Aspects of masticatory muscle stimulation: the myomonitor," Quitessence Int., vol. 3, pp. 57-58, 1972.

[19] U. S. Pal, L. Kumar, G. Mehta, N. Singh, M. Singh, and H. K. Yadav, "Trends in management of myofacial pain," Natl. J. Maxillofac. Surg., vol. 5, no. 2, pp. 109-116, 2014. 